\title{
Directional MAC Protocols in Ad-Hoc Networks
}

\author{
Sudipta Majumder \\ Department of CSE \\ Ahsanullah University of \\ Science \& Technology \\ Dhaka, Bangladesh
}

\author{
Syed Emdadul Haque \\ Department of EEE \\ Ahsanullah University of \\ Science \& Technology \\ Dhaka, Bangladesh
}

\author{
Fernaz Narin Nur \\ Senior Lecturer \\ Department of CSE \\ Daffodil International University \\ Dhaka, Bangladesh
}

\begin{abstract}
In wireless ad-hoc networks, the beam forming antenna technology is a new and promising solution to many challenges. Beam forming antennas have the ability to increase the spatial reuse, improve the transmission reliability, extend the transmission range and/or save the power consumption. If they are effectively used, they can significantly improve the network capacity, lifetime, connectivity and security. However, traditional Medium Access Control (MAC) protocols fail to exploit the potential benefits due to the unique characteristics of wireless ad-hoc networks with beam forming antennas. Ad-hoc networks suffer from the problem of hidden nodes (terminals), which leads to several degradation of network throughput. This survey gives a comprehensive overview of Medium Access Control (MAC) protocols which directly or indirectly address this problem. Open research discussions are also discussed to serve as a starting point for future protocol design and evaluation.
\end{abstract}

\section{Keywords}

Beam forming antennas, MAC protocol, Wireless ad-hoc network.

\section{INTRODUCTION}

Wireless networks are designed to provide single hop connectivity either to cellular base stations or to WLAN access points. However, the possibility of extending the wireless coverage, improving the overall capacity and enabling network auto-configuration with no infrastructure support has sparked the idea of multi-hop wireless networks [1]. IEEE 802.11 MAC is a carrier sense multiple accesses protocol with collision avoidance (CSMA/CA) mechanism which is used for several years to control the multiple access process of such networks. This protocol uses Omni-directional antennas for packet transmission. At this protocol, while two nodes are communicating with each other, the neighbor nodes have to stay silent. Due to the existence of silent area, the network losses a large portion of channel capacity and this leads to low network throughput particularly in dense networks. An Omni-directional antenna has a $360^{\circ}$ coverage angle. It sends signals towards all horizontal directions [20, 21]. However, since the energy is broadcasted in all directions and only a very small portion is actually received by the intended node, most of the energy transmitted is wasted [21]. Utilization of directional antennas is proposed in ad hoc networks to in order to compensate this problem.

A directional antenna can spread the energy to a certain direction, so it decreases the energy consumed by the transmitter. In addition, directional antennas have higher gains and greater transmission range consequently compared with omnidirectional antennas. By using directional antennas, a higher antenna gain can be achieved, which gives higher data rate and less transmission power. A directional antenna can increase the potential spatial reuse and network capacity $[19,20]$. In ad hoc networks with directional antennas the spatial reuse rate of wireless resources can be increased by configuring several nodes for interference free communication simultaneously[20,21]. In such a scenario, a potential link between two long-range nodes is established. The local congestion and end-to-end delay can be decreased by such long-range links [21]. Millimeter-wave communications also use directional antenna to combat severe path loss [23].

In the wireless ad-hoc network, as the channel is shared by all users, a medium access control (MAC) protocol is needed to coordinate different users to share the wireless channel fairly and resourcefully. The IEEE $802.11 \mathrm{DCF}$ is a widely used MAC protocol for wireless communications [21, 22]. IEEE 802.11 was designed for Omni-directional antennas, but its carrier senses multiple access with collision avoidance (CSMA/CA) is the foundation of many directional MAC protocol. However, using directional antennas in ad-hoc networks causes new challenges in MAC protocols such as new hidden terminal, deafness problems and unnecessary blocking of nodes. These problems basically occur when a transmitter does not have any information about the neighbor node's activities $[1,2,3]$. These problems result mainly from the fact that the data transmission period of certain nodes may overlap the RTS/CTS exchange period of their neighboring nodes or of nodes that are located outside the antenna beam of the RTS/CTS exchange. The smart beam forming antenna technology is promising technology to be utilized with multihop wireless networks [2,3]. Smart beam forming antennas have provided significant improvements in expanding coverage, mitigating interference and increasing capacity when deployed in cellular networks and wireless LANs [4, 5, 6]. However, Omni-directional antennas are dominating all forms of multi-hop wireless networks due to the cost and size limitations. On the other hand, the recent advances in the antenna technology along with the shift towards higher operating frequencies have made it feasible to use this technology even in small, mobile and battery-operated devices $[7,8]$. The lack of the appropriate control over the antenna beam forming may deteriorate the overall performance even below the level achieved by omnidirectional ones [9]. Hence, it is important to investigate innovative protocols, especially at the MAC layer, that are capable of harnessing the potential benefits of using smart beam forming antennas in wireless ad hoc networks.

\section{BEAM FORMING ANTENNA BASICS}

The primary function of any radio antenna is to couple electromagnetic energy from one medium to another. Traditionally, simple omnidirectional antennas are used to radiate/receive energy equally to/from all directions. On the other hand, directional antennas are able to radiate/receive energy to/from one direction more than the others. Since the 
transmission and reception characteristics of the antenna are reciprocal, the directional antenna has both transmission and reception gains. A directional antenna pattern usually consists of a high gain main lobe and smaller gain side and back lobes $[18,19,20]$. Since a directional antenna is able to radiate energy in the direction of the intended receiver, this transmission does not interfere with neighboring nodes residing in other directions.

For the same transmit power as Omni-directional antennas, the directional gain of beam forming antennas is translated to communication range extension. This extension may lead to fewer-hops routes and consequently a reduction in the end-toend delay [25]. In addition, the communication range extension makes it possible to bridge network partitions [26] and may improve the connectivity of the network [27]. The unique features of beam forming antennas reduce the risks of eavesdropping and jamming, hence, providing more secure wireless communication [28,29].

A directional antenna is thought of to be an isotropic antenna with a constant gain over a certain angle $\Omega_{\mathrm{A}}$, as shown in fig1. For the directional mode, the antenna directional pattern that we adopt is that there is a constant gain in the main lobe and we do not consider the interference created by the side lobes. According to $[18,19]$ the area size of the side lobes is much smaller than the area size of the main lobes.

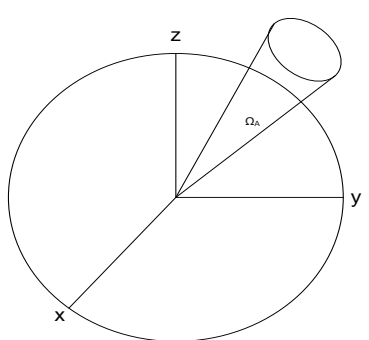

Fig 1: Antenna Pattern

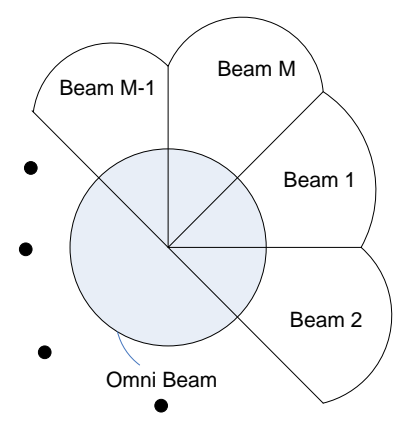

Fig 2: Antenna Model
So, the probability of finding wireless nodes within the side lobes of the antenna is very small and can be ignored. When the node does not know the direction that signal may arrive from, the node is in the omnidirectional mode, and the omnidirectional gain is $\mathrm{G}_{0}$. Once the signal is detected, the antenna uses the direction of arrival (DOA) algorithm to determine the DOA, and goes into the directional mode. Mainly, there are three types of directional antenna systems: switched beam antenna system, steered beam antenna system, and adaptive antenna system [21].

The antenna system possesses two separate modes: Omni and Directional. In Omni mode, a node receives signals from all directions with gain $\mathrm{G}^{0}$. Initially an idle node waits for signals in Omni mode. After a signal is sensed in Omni mode, the antenna detects the beam to specified direction on which the signal power is strongest and goes into the Directional mode. In directional mode a node can point its beam towards a specific direction with gain $G^{d}>G^{0}[21,22,23]$. Beam forming antennas are classified into switched beam systems and steered beam systems.

\subsection{Switched Beam Antenna Systems}

In switched beam systems, the antenna array is combined with a fixed Beam Forming Network (BFN). The BFN consists of a predetermined set of weight vectors, where the configuration of weights in a vector determines the direction in which the antenna radiation pattern is beam formed.
Switched beam antennas can provide most of the benefits of smart antennas at a small fraction of complexity and expense. Spatial reuse, range extension and power saving are possible with this type of smart antennas. However, they do not guarantee maximum gain due to scalloping [4]. Scalloping is the roll-off of the antenna pattern as a function of the angle from the bore sight.

\subsection{Steered Beam Antenna Systems}

They are also known as Adaptive Antenna Array (AAA) systems. They provide a high degree of flexibility in configuring the radiation patterns. Using a variety of sophisticated signal processing algorithms, the adaptive array antennas can adapt their weights in order to maximize the resulting Signal to Interference and Noise Ratio (SINR). The bore sight of the main lobe can be directed towards the target using phase shifters. This type is known as phased antenna arrays. Although adaptive antenna array systems can outperform switched beam systems especially in multi-path environments, the associated complexity and cost are limiting factors [14]. The need to continuously locate and track various types of signals complicates the signal processing task and results in a significant increase in the power consumption.

\section{MAC PROTOCOL BASED ON DIRECTIONAL ANTENNA}

Directional antenna-based protocols allow for simultaneous data transmission and reception in order to increase spatial reuse. They minimize the probability of frame collisions (also caused by hidden nodes) and usually achieve higher network efficiency than IEEE 802.11. In MAC protocols using directional antenna, simultaneous data transmission and reception increases spatial reuse, minimized probability of collisions, can take advantage of multiple channels, higher network efficiency than IEEE 802.11. But new kinds of hidden nodes, higher directional interference and deafness, performance decreases in directional MAC protocols with node mobility, additional hardware complexity, in most cases large signaling overhead and slow channel reservation, performance strongly dependent on network topology, performance can be deteriorated by the side lobe problem.

\subsection{Challenges with Directional Antenna}

Conventional wireless MAC protocols were designed to overcome the challenges of the wireless medium such as hidden terminal problem and exposed terminal problem. The unique characteristics of directional antennas pose unparalleled challenges that should be considered in the design of the directional MAC protocols for wireless ad hoc networks. In this section, we discuss the main challenges facing the medium access control in directional MAC protocol.

\subsubsection{Deafness}

While exploiting the spatial reusability using beam forming antennas, deafness is by far the most critical challenge [30, 31]. Deafness [32] was first identified in the context of the Basic Directional MAC (DMAC) protocol [10]. It occurs when a transmitter tries to communicate with a receiver but fails because the receiver is beam formed towards a direction away from the transmitter. 


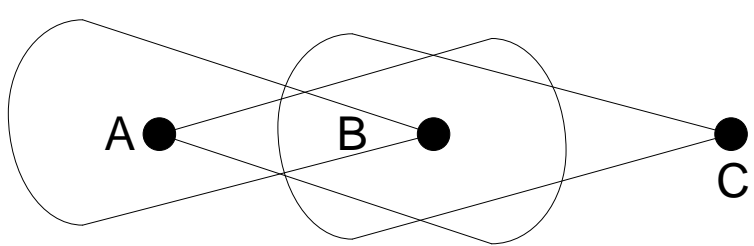

Fig3: Deafness Scenario

In fig3 node A and B is communicating with each other. Node $\mathrm{C}$ is trying to initialize communication with node $\mathrm{B}$ where node $\mathrm{B}$ is beam formed to node $\mathrm{A}$. For that, node $\mathrm{C}$ will not get DCTS in time and double its back off time for retransmission, as it concludes that a collision has occurred. When node $\mathrm{C}$ reaches its retry limit, it concludes that node $\mathrm{B}$ is unreachable. If the MAC protocol requires the node to carrier sense, back off and communicate directionally, it may suffer from prolonged period of deafness if it has multiple back-to-back packets to be transmitted. Moreover, a chain of deafness is also possible in which each node attempting to communicate with a deaf node becomes itself deaf to another node. This could also result in a deadlock scenario [33].

\subsubsection{Hidden Terminal Problem}

The traditional hidden terminal problem in wireless networks occurs when two nodes are outside the carrier sensing range of each other and both of them attempt to communicate with a common node causing collision. The collision avoidance concept was proposed to solve this problem which is implemented by means of RTS/CTS handshaking before data transmission [33]. In the context of directional antennas, the hidden terminal problem occurs when a potential interferer could not receive the RTS/CTS exchange due to its antenna orientation during the handshake and then initiates a transmission that causes collision. In other word, hidden terminal problems arise when the transmitter and receiver have not heard the DRTS or DCTS.

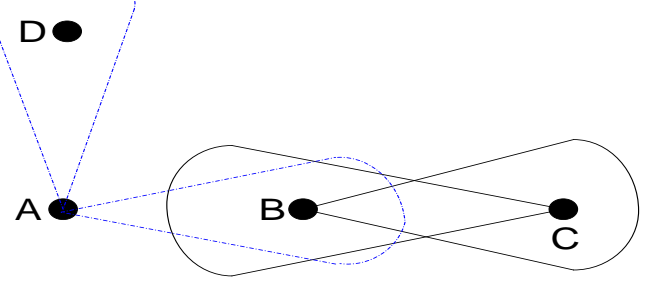

Fig4: Hidden Station Scenario

For illustration, in Fig4, while node B is sending DRTS to node $\mathrm{C}$, node A is busy in communication with D. Node C's DCTS cannot reach to node A, as it is beam formed in the direction of $\mathrm{D}$. When the communication between nodes $\mathrm{B}$ and $\mathrm{C}$ is in progress, if has finished its transmission to $\mathrm{D}$ and has a packet to send to node B. At that time A initiates communication with B by sending DRTS to B, and it will lead to a collision with B's transmission. Hidden terminals can severely degrade the network performance. Regrettably, the standard RTS/CTS mechanism fails to completely solve the problem, as nodes near to the source node may initiate transmissions during the time the source node transmits the RTS.

\subsubsection{Asymmetry in Gain Problem}

When a directional transmission for data packets and an Omni-directional transmission for control packets such as RTS/CTS are used, different transmission ranges lead to the asymmetry-in-Gain problem because the transmission range of a directed signal and the transmission range of an Omnidirectional signal are not identical.

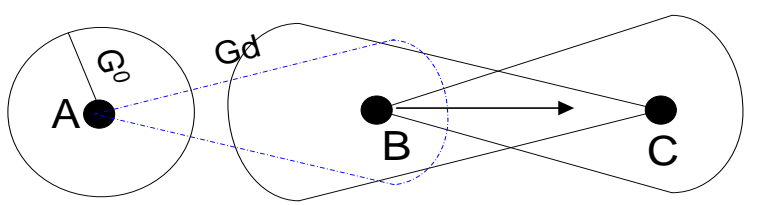

Fig5: Asymmetry in gain scenario

In Fig5, when the nodes are in idle mode they listen Omnidirectionally with a gain of G0. For example, B initiates transmission to node $\mathrm{C}$ by sending DRTS. Then node $\mathrm{C}$ sends DCTS to node B. At that moment node A is in Omnidirectional mode as it is in idle state. Node A is far enough away from node C, so A cannot hear the DCTS from C because the Omni-directional gain G0 is smaller than the directional gain $\mathrm{Gd}$. When nodes $\mathrm{B}$ and $\mathrm{C}$ begin data transmission by pointing their transmission and reception beams to each other with a gain $\mathrm{Gd}$, at the same time A also wants to communicate with B using DRTS which interfere the ongoing data transmission.

\subsubsection{HOL Blocking Problem}

The Head-of-Line (HOL) blocking problem with directional MAC protocols was first acknowledged in [34]. It occurs as a result of the typically used First-In-First-Out (FIFO) queuing policy. This policy works fine in the presence of omnidirectional antennas since all outstanding packets use the same medium. If the medium is busy, no packets can be transmitted. If the channel in the outgoing direction of the head of the RTS queue is in use, the node will have to wait until the channel is available to send the head RTS packet. In fact, during the blocking time, the channels in the outgoing directions of other RTS packets in the RTS queue are idle probably. Thus, the head of RTS queue blocks the transmission of other packets, which is the head-of-line blocking problem.

\subsection{Directional MAC Protocols}

The predicament of designing an efficient MAC protocol for wireless ad hoc networks with directional antennas has been of a great interest during the last decade. In this section, we present portrayal of the proposed directional MAC protocols. The MAC protocols can be roughly classified into random access protocols and synchronized access protocols. Random access protocols consent to the stations to access the shared medium randomly through contention with each other. Synchronized access protocols consent to the stations to access the medium based on a predetermined schedule which can be achieved through local and/or global synchronization.

\subsubsection{DMAC}

The basic Directional MAC (DMAC) protocol assumes that the higher layers are aware of neighbors of a particular node and are able to provide transceiver profiles required to communicate with each neighbor [10]. The operation of DMAC is based on the four-way handshake mechanism. Nodes listen on the wireless channel Omni-directionally and create directional NAV (DNAV) tables to keep track of ongoing transmission directions and their corresponding durations. Channel reservation is done with directionally transmitted DRTS and DCTS frames preceded by the Back off procedure. DATA and ACK frames are also sent directionally. The authors of DMAC admit that using directional RTS/CTS transmissions introduces two new kinds 
of hidden nodes - hidden nodes caused by asymmetry in gain and hidden nodes caused by unheard RTS/CTS. The deafness problem appears when a node does not hear RTS/CTS exchanges for new transmissions because it is engaged in its own directional transmission.

The problem of node deafness is addressed in [11]. A variation of this protocol is DMAC with Power Control and Directional Receiving (DMAC-PCDR) [12]. The authors of DMAC-PCDR assume that each node is equipped with GPS and the location information is obtained at the MAC layer. Idle nodes rotate the directional receiving antenna beams, which solves the directional hidden node problem. Finally, the protocol supports two transmission power controls in order to reduce the interference range and increase spatial reuse of the wireless channel.

\subsubsection{CDR-MAC}

The Circular Directional RTS MAC (CDR-MAC) protocol supports neighbor discovery and tracking [13]. CDR-MAC introduces consecutive directional transmission of RTS frames in directions to inform neighbors about its planned transmission. The circular directional transmission is achieved thanks to antennas with a predefined number of beams. After overhearing the RTS frame, the destination node transmits a directional CTS frame for which the sender listens Omni directionally. The transmissions of DATA and ACK frames are also directional. To resolve the problem of neighbor's locations each node maintains a Location Table, with information about beams from which the transmitter received a frame and by which the destination node transmitted a frame. The Location Table is updated every time a node receives a frame. Thanks to the circular directional transmissions of RTS frames and Location Tables the protocol alleviates the hidden node and the deafness problems. The performance of CDR-MAC can be, however, degraded by long DIFS intervals, RTS collisions and interference from minor lobes [14]. Additionally, CDR-MAC has a large signaling overhead.

\subsubsection{BMAC}

The BMAC [35] protocol for ad hoc networks with adaptive antenna arrays in. BMAC performs joint channel gathering and medium sharing. The channel acquisition is performed proactively through a periodic training sequence. When receiving this training sequence, the channel to the corresponding node is estimated and the channel coefficients and the node identifier are saved in a channel table. When there is data ready to be sent, the source node sends a beam formed RTS (BRTS) to maximize the power at the destinations and make nulls towards the potentially interfering neighbors. When receiving the BRTS, the destination node calculates the exceeded power for further transmitted power correction and then it sends an Omni-directional CTS (OCTS) packet containing this correction factor. The results show that BMAC offers higher throughput than the conventional DMAC in multi-path fading environment.

\subsubsection{CDMAC}

The Coordinated Directional MAC (CDMAC) [15] allows for parallel directional DATA/ACK transmissions. Additionally, it uses Omni-directional RTS/CTS transmissions to minimize the deafness problem. It is assumed that each node has a single transceiver but it can dynamically switch from directional to Omni-directional transmission and reception.
Parallel DATA/ACK transmissions are possible thanks to a coordinated MAC timing-structure in which, in order to reserve the wireless channels and silence possible hidden nodes, multiple Omni-directional RTS/CTS frame exchanges take place before any DATA transmission. The RTS/CTS/CRTS handshake is used for both Slave Node (SN) and Master Node (MN) contention. Additionally, similarly to DMAC, each node maintains a DNAV table, which is checked during the probing phase and is updated during the beam-indication phase. This way of two-step channel reservation allows reducing the interference among nodes.

\subsubsection{DMAC-DACA}

DMAC protocol with Deafness Avoidance and Collision Avoidance (DMAC-DACA) protocol [35] is the basic directional RTS/CTS exchange is followed by sweeping RTS/CTS counterclockwise to inform all the neighbors about the upcoming communication. Deafness is avoided using a deafness neighbor table that uses the sweeping RTS/CTS to record the deafness duration of neighboring nodes. The location information, retrieved by GPS, is added to the RTS/CTS frames. Using this information, the node that receives RTS/CTS can update the record in its deafness neighbor table if any of the neighbors is in the coverage area of the upcoming transmission. The DMAC-DACA protocol performs collision avoidance through the DNAV mechanism.

\subsubsection{MCDA}

In the Multi-Channel MAC protocol with a Directional Antenna (MCDA) [16] each node is equipped with a single antenna and has to switch between the data and control channels. Therefore, to cope with the channel collision problem, here situation in which the control-channel information cannot be heard because a node is tuned to a data channel. This protocol adopts the channel switch sequence (CSS) mechanism. Additionally, MCDA implements RTS/CTS/DATA/ACK exchange. All CTS/DATA/ACK frames are transmitted directionally. MCDA operates in three phases: negotiation, communication, and block. In the first phase the source-destination pair exchanges RTS/CTS frames on the control channel to select the data channel. In the communication phase, the nodes exchange RTS/CTS frames on the selected data channel to avoid the hidden node problem. The block phase is used in overload situations. If a pair of communicating nodes stays in the block phase it switches to a single data channel and waits for the channel to become idle.

\subsubsection{MARS}

Multiple Access Scheme with Sender driven and Reception first for Smart antenna (MARS) [17] is a protocol which changes RTR frames into Ready to Receive and Transmit (RTRT) frames. RTRT frames include addresses of the source and destination nodes. A node transmits an RTRT frame when it has data to send. Because the RTRT frame includes the transmitter address, the neighbors of the middle node can adjust their antennas to its direction and transmit their RTS frames. If a particular neighboring node does not have data to send it sets the size of data to zero. Upon reception of the RTS frames the middle node adjusts the gain pattern of its antenna and transmits all corresponding CTS frames. This way of operation allows reducing polling overhead and interference in comparison to the IEEE 802.11 standard. 


\subsubsection{PMAC}

A synchronous Polling-based MAC (PMAC) protocol for mobile ad hoc networks with directional antennas [36]. In this protocol, the time is divided into contiguous frames and each frame is divided into three segments: search, polling and data transfer. In the search segment, each node searches for new neighbors by transmitting or receiving pilot tones directionally. If two nodes discover each other, they exchange a list of the available slots in their corresponding polling segments. Once a pair of nodes agree upon a polling slot, they communicate in the same slot periodically, frame after frame, until they lose connectivity. The communication in the polling slot is preceded by the exchange of control packets to avoid collisions. In PMAC, RTS and CTS messages are used prior to the data transfer in order to detect possible rare collisions. The results show that PMAC achieves high channel utilization even in mobile scenarios.

\subsubsection{DSDMAC}

Dual-sensing directional MAC (DSDMAC) protocol [37] relies on the dual-sensing strategy to identify deafness, resolve the hidden-terminal problem, and avoid unnecessary blocking. The protocol verification, analysis, and simulation results show the robustness and superior performance of the DSDMAC protocol, which can achieve a much higher network throughput and lower delay utilizing the spatial multiplexing gain of the directional antennas. The proposed DSDMAC protocol uses two well-separated wireless channels, i.e., a data channel and a busy-tone channel. The data channel carries the data packets and the RTS, CTS, and ACK packets on a specified direction (DRTS, DCTS, DDATA, and DACK). On the other hand, the busy-tone channel will be used to transmit a sine-wave busy-tone signal on all other directions. The protocol assumes that the directions of all reachable destinations or forwarders are predetermined. The integrity of the DSDMAC has been verified using a formal protocol verification tool called Spin. Spin results have shown that the DSDMAC protocol outperforms the existing protocols.

\subsubsection{RDMAC}

In [24], Reservation Directional MAC (RDMAC) is proposed for multi-hop wireless networks with directional antennas. The RDMAC protocol operates in sessions with each session comprising a reservation period and a transmission period. In the reservation period, the first node to transmit the RTS defines the start and end time of the transmission period. Each node-pair exchanges four control packets. The neighboring nodes that receive the ORTS/OCTS packets estimate the direction of arrival and point their antennas towards the sender/receiver to receive the remaining control packets. The reserving nodes transmit directional RTS/CTS packets so the neighbor nodes can update their DNAV taking into consideration any possible interference caused by minor lobes.

\section{MAIN COMPARISON BETWEEN PROTOCOLS}

In the previous sections, we classified the existing MAC approaches for wireless ad-hoc networks with beam forming Antennas and briefly discussed the protocols. In this section, we provide a comparative summary of the reviewed MAC protocols.

Table 1. Summarize the main features of the MAC protocol

\begin{tabular}{|c|c|c|c|c|c|c|}
\hline $\begin{array}{l}\text { Protocol } \\
\text { Name }\end{array}$ & $\begin{array}{l}\text { Required change for } \\
\text { modification }\end{array}$ & $\begin{array}{l}\text { Reservation } \\
\text { method }\end{array}$ & Access Type & Hardware & Overhead & QoS \\
\hline DMAC & $\begin{array}{l}\text { Frames are transmitted using } \\
\text { directional antennas, medium is } \\
\text { sensed using Omni-directional } \\
\text { antennas, each node maintains a } \\
\text { directional NAV table. }\end{array}$ & RTS/CTS & $\begin{array}{l}\text { Random } \\
\text { Access(Rely } \\
\text { solely on } \\
\text { control packets) }\end{array}$ & $\begin{array}{l}\text { Complex } \\
\text { (directional } \\
\text { transmission, omni- } \\
\text { directional reception) }\end{array}$ & Large & No \\
\hline BMAC & $\begin{array}{l}\text { It is channel-based and uses the } \\
\text { adaptive beam forming } \\
\text { techniques and not conventional } \\
\text { directional antenna. }\end{array}$ & BRTS/CTS & $\begin{array}{l}\text { Random } \\
\text { Access(Rely } \\
\text { solely on } \\
\text { Control packets) }\end{array}$ & $\begin{array}{l}\text { Complex } \\
\text { (directional antennas, } \\
\text { rotation of receiving } \\
\text { antenna beams) }\end{array}$ & Large & Yes \\
\hline $\begin{array}{l}\text { DMAC- } \\
\text { PCDR }\end{array}$ & $\begin{array}{l}\text { Each node equipped with GPS, } \\
\text { smart usage of Omni-directional } \\
\text { and directional antennas, } \\
\text { rotation of receiving antenna } \\
\text { beams. }\end{array}$ & RTS/CTS & $\begin{array}{l}\text { Random Access } \\
\text { (With tone) }\end{array}$ & $\begin{array}{l}\text { Complex } \\
\text { (directional } \\
\text { antennas, rotation } \\
\text { of receiving } \\
\text { antenna beams) }\end{array}$ & Large & No \\
\hline CDR-MAC & $\begin{array}{l}\text { Circular directional transmission } \\
\text { of RTS, directional antennas } \\
\text { with predefined number of } \\
\text { beams, multiple RTS } \\
\text { transmissions, each node } \\
\text { maintains location table. }\end{array}$ & RTS/CTS & Random Access & $\begin{array}{l}\text { Complex } \\
\text { (directional } \\
\text { transmission) }\end{array}$ & $\begin{array}{l}\text { Very } \\
\text { large }\end{array}$ & No \\
\hline RDMAC & $\begin{array}{l}\text { Smart usage of directional and } \\
\text { Omni-directional antennas, } \\
\text { additional signaling. }\end{array}$ & RTS/CTS & $\begin{array}{l}\text { Synchronized } \\
\text { Access }\end{array}$ & $\begin{array}{l}\text { Complex (directional } \\
\text { and omni-directional } \\
\text { transmission) }\end{array}$ & Large & No \\
\hline $\begin{array}{l}\text { DMAC- } \\
\text { DACA }\end{array}$ & $\begin{array}{l}\text { Smart usage of directional and } \\
\text { Omni-directional antennas. }\end{array}$ & RTS/CTS & Random Access & Complex & Large & No \\
\hline PMAC & $\begin{array}{l}\text { Smart usage of directional } \\
\text { antennas. }\end{array}$ & $\begin{array}{l}\text { DRTS/ } \\
\text { DCTS }\end{array}$ & $\begin{array}{l}\text { Synchronized } \\
\text { Access }\end{array}$ & Complex & Large & No \\
\hline
\end{tabular}




\begin{tabular}{|c|c|c|c|c|c|c|}
\hline CDMAC & $\begin{array}{l}\text { parallel directional DATA/ACK } \\
\text { transmissions, Omni directional } \\
\text { transmissions of RTS/CTS, each } \\
\text { node has a single transceiver and } \\
\text { maintain a DNAV table. }\end{array}$ & $\begin{array}{l}\text { RTS/CTS/ } \\
\text { CRTS }\end{array}$ & $\begin{array}{l}\text { Synchronized } \\
\text { Access }\end{array}$ & $\begin{array}{l}\text { Complex } \\
\text { (directional and } \\
\text { omni-directional } \\
\text { transmission) }\end{array}$ & Low & No \\
\hline MCDA & $\begin{array}{l}\text { Smart usage of directional } \\
\text { antennas, It adopts the channel } \\
\text { switch sequence (CSS) } \\
\text { mechanism. }\end{array}$ & RTS/CTS & Random Access & $\begin{array}{l}\text { Complex } \\
\text { (directional antennas) }\end{array}$ & Large & No \\
\hline DSDMAC & $\begin{array}{l}\text { Dual-sensing directional } \\
\text { antennas, omnidirectional } \\
\text { transmissions of RTS/CTS, } \\
\text { directions of all reachable } \\
\text { destinations or forwarders are } \\
\text { predetermined. }\end{array}$ & $\begin{array}{l}\text { DRTS / } \\
\text { DCTS }\end{array}$ & Random Access & $\begin{array}{l}\text { Complex } \\
\text { (dual-sensing } \\
\text { directional antennas) }\end{array}$ & Large & No \\
\hline MARS & $\begin{array}{l}\text { Additional signaling (ready-to- } \\
\text { receive and transmit frame), } \\
\text { changed RTS frame format, } \\
\text { smart antennas. }\end{array}$ & $\begin{array}{l}\text { RTRT/RTS/ } \\
\text { CTS }\end{array}$ & $\begin{array}{l}\text { Synchronized } \\
\text { Access }\end{array}$ & $\begin{array}{l}\text { Complex } \\
\text { (Smart antennas) }\end{array}$ & Very Large & No \\
\hline
\end{tabular}

The existing MAC protocols for wireless ad-hoc networks with beam forming antennas differs in terms of the supported communication range for a specific transmission power. The early proposals for directional MAC have aimed to exploit the benefits of beam forming antennas through the direct adaptation of existing protocols such as IEEE 802.11. Later on, several unprecedented beam forming related challenges have been identified and the directional MAC designers have focused on proposing mechanisms to solve these new problems. Different mechanisms have been proposed to cope with these problems on the expense of benefits offset and/or additional complexity. In this paper, we compare the advantages and disadvantages of the different directional MAC design choices proposed in the literature. The features of each protocol include the transmission of the basic protocol packets, the antenna mode during idle listening, the transmission of tones in some cases, the back off mechanism, the number of channels required, whether power control is employed or not, the type of the antenna and its capabilities, the supported communication range, how the MAC protocol acquires beam forming information and finally the main directional MAC challenges addressed by each protocol. Almost all the proposed MAC protocols exchange DATA and ACK packets directionally to exploit the benefits of directional antennas. However, there are many variations in the transmission of RTS/CTS packets. The omnidirectional transmission of RTS/CTS is beneficial to inform all neighbors about imminent communication and consequently, reduce the instances of deafness and hidden terminal problem significantly. To enable more simultaneous transmissions and extended range communications, several MAC protocols have used directional RTS/CTS handshakes assuming the beam forming information is known a priori which is indeed a challenging task. Moreover, this aggressive reservation scheme lends itself to deafness and directional hidden terminal problems. To address the fundamental tradeoff between Omni-directional RTS/CTS and uni-directional RTS/CTS, some MAC protocols rely on the multi-directional RTS/CTS for channel reservation. With switched beam antennas, the control packets are transmitted circularly, one direction after the other, to allow for collision and/or deafness avoidance. The main drawback of this scheme is its large control overhead that can sometimes offset the benefits [33]. Few MAC protocols transmit the RTS/CTS packets concurrently to multiple directions using sophisticated adaptive antenna array systems that can form multi-beam radiation patterns. Some directional MAC protocols use tones instead of the circular RTS/CTS packets to address the MAC challenges. Tones are commonly transmitted on a dedicated control channel which offsets the bandwidth. Tones are usually transmitted directionally to protect ongoing communication from collisions but transmitted Omnidirectionally to announce the start and/or the end of a communication to handle the deafness problem. The drawback of tone-based protocols is the additional required hardware that increases cost and complexity. Another approach to handle the MAC challenges is to perform synchronized access rather than random access. By separating the transfer of control and data packets in time, the locationdependent carrier sensing problems are alleviated. Time is divided into frames with the data transfer sub frame is preceded with a contention-based reservation sub frame. Early synchronized directional MAC protocols assume the availability of network-wide synchronization which is challenging to achieve in multi-hop wireless networks. Few recent synchronized MAC schemes are based on local synchronization. The first winning node-pair in the contention based period decide the size of control and data sub-frames. Although this approach alleviates the complexity of global synchronization, setting the size of the control and data sub frame is a critical tradeoff between under-utilization and poor spatial reuse. Moreover, communication between certain neighboring node-pair may not be properly scheduled if each of them lies in a different synchronized zone. Although the binary exponential back off (BEB) mechanism was originally designed for networks with Omni-directional antennas. The DATA transmission power is adjusted based on the information collected using the RTS/CTS packets in most protocols. Throughout the literature, different beam forming antenna types have been used in wireless ad-hoc networks. Switched beam antennas are the most used since they are the simplest type and hence facilitating the development of directional MAC protocols. However, some MAC protocols are proposed based on the use of steered beam antennas. 


\section{FUTURE RESEARCH DIRECTIONS}

Significant research has already been performed in the area of MAC protocol in wireless ad-hoc network. However, a number of issues remain unresolved or not completely addressed. Designing a new protocol resilient to hidden nodes and deaf nodes require careful consideration. This is because the problem of hidden nodes and deaf nodes can be seen from several different angles. This problem also appears in cognitive networks, which are currently receiving increased attention. Additionally, if directional antennas are used, some new kinds of hidden nodes may appear. Finally, to our best knowledge in the literature there is no survey which elaborates on the complexity of the problems in detail. Designing a new protocol resilient to hidden nodes and deafness nodes requires careful consideration. This is because the problems can be seen from several different angles. New protocols should additionally avoid starvation. Furthermore, not all protocol described in this paper support Quality of Service (QoS). Therefore, it can be predictable that future wireless networks will require QoS-aware solutions. Ideal solutions require QoS support, admission control, traffic policing, adequate bandwidth allocation and reservation, etc. Again, another important issue is fairness. Studies have shown that the current IEEE 802.11 standard does not assure a fair share of resources if hidden nodes are present in the network. Therefore, future research should focus on the problem of fairness in multi-hop networks. Additionally, the proposed solutions are in most cases not tested in real environments. Therefore, future studies should rather be devoted to real implementations than just simulations. Only such an approach can ultimately verify a protocol's usefulness in future wireless networks.

\section{CONCLUSION}

In this paper we have discussed various methods and techniques used so far in the design and development of wireless ad hoc network. We also looked into antenna technologies used especially the directional or the beam forming antennas. This was done to give a straightforward view on the progress made in the area of MAC protocols. The latter aimed at speculating about expected challenges to give research ideas to future protocol designers.

\section{REFERENCES}

[1] C. E. Perkins, Ad Hoc Networking. Addison-Wesley, 2001.

[2] R. Ramanathan, "On the Performance of Ad Hoc Networks with Beamforming Antennas," in ACM International Symposium on Mobile Ad Hoc Networking and Computing (MobiHoc), Long Beach, California, October 2001, pp. 95-105.

[3] J. H. Winters, "Smart Antenna Techniques and Their Application to Wireless Ad Hoc Networks," IEEE Wireless Commun., vol. 13, no. 4, pp. 77-83, August 2006.

[4] J. C. Liberti and T. S. Rappaport, Smart Antennas for Wireless Communications. Prentice Hall, NJ, 1999.

[5] L. Godara, "Applications of Antenna Arrays to Mobile Communications, Part I: Performance Improvement, Feasibility and System Considerations," Proc. IEEE, vol. 85, no. 7, pp. 1031-1060, July 1997.
[6] A. Doufexi, S. Armour, A. Nix, P. Karlsson, and D. Bull, "Range and Throughput Enhancement of Wireless Local Area Networks Using Smart Sectorised Antennas," IEEE Trans. Wireless Commun., vol. 3, no. 5, pp. 1437-1443, September 2004.

[7] D. Leang and A. Kalis, "Smart SensorDVBs: Sensor Network Development Boards with Smart Antennas," in IEEE International Conference on Communications, Circuits and Systems (ICCCAS), Chengdu, China, June 2004, pp. 1476-1480.

[8] G. Giorgetti, A. Cidronali, S. K. Gupta, and G. Manes, "Exploiting Low-Cost Directional Antennas in $2.4 \mathrm{GHz}$ IEEE 802.15.4 Wireless Sensor Networks," in European Conference on Wireless Technologies, Munich, Germany, October 2007, pp. 217-220.

[9] R. Ramanathan, "Antenna beamforming and power control for ad hoc networks," Book chapter on Mobile Ad Hoc Networking, Wiley-IEEE Press, pp. 139-174, 2004.

[10] R.R. Choudhury, X. Yang, R. Ramanathan, N.H. Vaidya, Using directional antennas for medium access control in ad hoc networks, in: Proc. of the 8th ACM Annual International Conference on Mobile Computing and Networking, 2002, pp. 59-70.

[11] R.R. Choudhury, N.H. Vaidya, Deafness: A MAC problem in ad hoc networks when using directional antennas, in: Proc. of the 12th IEEE International Conference on Network Protocols, 2004, pp. 283-292.

[12] Y. Takatsuka, M. Takata, M. Bandai, T. Watanabe, A MAC protocol for directional hidden terminal and minor lobe problems, in: Wireless Telecommunications Symposium - WTS, 2008, pp. 210-219.

[13] T. Korakis, G. Jakllari, L. Tassiulas, CDR-MAC: a protocol for full exploitation of directional antennas in ad hoc wireless networks, IEEE Transactions on Mobile Computing (2007) 145-155.

[14] J.J. Chang, W. Liao, T.C. Hou, Reservation-based directional medium access control (RDMAC) protocol for multi-hop wireless networks with directional antennas, in: Proc. of the IEEE International Conference on Communications - IEEE ICC, 2009, pp. 4898-4902.

[15] J.Wang, H. Zhai, P. Li, Y. Fang, D.Wu, Directional medium access control for ad hoc networks, Wireless Networks 15 (8) (2009) 1059-1073.

[16] C.Y. Chang, Y.C. Chen, L.L. Hung, S.W. Chang, A novel multi-channel MAC protocol with directional antenna for enhancing spatial reuse and bandwidth utilization in WLANs, Journal of Parallel and Distributed Computing 69 (10) (2009) 824-837.

[17] W.K. Lai, K.S. Tseng, J.C. Chen, MARS: a multiple access scheme with sender driven and reception first for smart antenna in ad hoc networks, Wireless Communications and Mobile Computing 9 (2) (2009) 197-208.

[18] C. A. Balanis, Antenna Theory: Analysis and Design. Wiley, NY, 1997.

[19] L. Godara, "Applications of Antenna Arrays to Mobile Communications, Part II: Beam-Forming and Directionof-Arrival Considerations," Proc. IEEE, vol. 85, no. 8, pp. 1195-1245, August 1997. 
[20] C. A. Balanis and P. Ioannides, Introduction to Smart Antennas. Morgan and Claypool Publishers, 2007.

[21] Lichun Bao and J. J. Garcia-Luna-Aceves,"Receiveroriented multiple access in ad hoc networks with directional antennas. Wirel. Netw.,11(1-2):67-79, January 2005.

[22] Young-Bae Ko, V. Shankarkumar, and N.F.Vaidya. Medium access control protocols using directional antennas in ad hoc networks. In INFOCOM 2000. Nineteenth Annual Joint Conference of the IEEE Computer and Communications Societies. Proceedings. IEEE, volume 1, pages 13-21vol.1, 2000.

[23] Ram Ramanathan. On the performance of ad hoc networks with beamforming antennas. In Proceedings of the 2Nd ACM International Symposium on Mobile Ad Hoc Networking \&Amp; Computing, MobiHoc '01, pages $95\{105$, New York, NY, USA,2001. ACM.

[24] S. L. Karthikeyan Sundaresan and R. Sivakumar, "On the Use of Smart Antennas in Multi-Hop Wireless Networks," in IEEE International Conference on Broadband Communications, Networks and Systems, San Jose, California, October 2006, pp. 1-10.

[25] R. Choudhury and N. Vaidya, "Performance of Ad Hoc Routing using Directional Antennas," Elsevier Journal of Ad Hoc Networks, vol. 3, no. 2, pp. 157-173, March 2005.

[26] A. K. Saha and D. B. Johnson, "Routing Improvement using Directional Antennas in Mobile Ad Hoc Networks," in IEEE Global Telecommunications Conference (GLOBECOM), vol. 5, Dallas, Texas, November 2004, pp. 2902-2908.

[27] P. Li, C. Zhang, and Y. Fang, “Asymptotic Connectivity in Wireless Ad Hoc Networks Using Directional Antennas," IEEE/ACM Trans. Netw., vol. 17, no. 4, pp. 1106-1117, August 2009.

[28] G. Noubir, "On Connectivity in Ad Hoc Network under Jamming Using Directional Antennas and Mobility," in International Conference on Wired /Wireless Internet Communications, 2004, pp. 521-532.

[29] M. Zefreh and P. Khadivi, "Secure Directional Routing to Prevent Relay Attack," in International Conference on Information and Communication Technologies: from
Theory to Applications, Damascus, Syria, April 2008, pp. $1-6$.

[30] H. Gossain, C. Cordeiro, D. Cavalcanti, and D. P. Agrawal, "The Deafness Problems and Solutions in Wireless Ad Hoc Networks using Directional Antennas," in IEEE Global Telecommunications Conference (GLOBECOM) Workshops, November 2004, pp. 108114.

[31] M. Takata, M. Bandai, and T. Watanabe, "A MAC Protocol with Directional Antennas for Deafness Avoidance in Ad Hoc Networks," in IEEE Global Telecommunications Conference (GLOBECOM), Washington, USA, November 2007, pp. 620-625.

[32] R. Choudhury and N. Vaidya, "Deafness: A MAC Problem in Ad Hoc Networks when Using Directional Antennas," in IEEE International Conference on Network Protocols (ICNP), Berlin, Germany, October 2004, pp. 283-292.

[33] S. S. V. Bharghavan, A. Demers and L. Zhang, "MACAW: A Media Access Protocol for Wireless LANs," in ACM International Conference of the Special Interest Group on Data Communication (SIGCOMM), London,UK, August 1994, pp. 212-225.

[34] V. Kolar, S. Tilak, and N. B. Abu-Ghazaleh, "Avoiding Head of Line Blocking in Directional Antenna," in IEEE International Conference on Local Computer Networks $(L C N)$, Zurich, Switzerland, November 2004, pp. 385392.

[35] K. Fakih, J.-F. Diouris, and G. Andrieux, "Beamforming in Ad Hoc Networks: MAC Design and Performance Modeling," EURASIP Journal on Wireless Communications and Networking, vol. 2009, Article ID 839421, 15 pages, 2009. doi:10.1155/2009/839421.

[36] G. Jakllari, W. Luo, and S. V. Krishnamurthy, "An Integrated Neighbor Discovery and MAC Protocol for Ad Hoc Networks Using Directional Antennas," IEEE Trans. Wireless Commun., vol. 6, no. 3, pp. 11-21, March 2007.

[37] Abdullah, A.A., Lin Cai and Gebali, F., "DSDMAC: Dual Sensing Directional MAC Protocol for Ad Hoc Networks with Directional Antennas," IEEE Trans. On Vehicular Technology ,vol. 61, no. 3, March 2012,PP. $1266-1275$. 